\title{
Salvage Surgery and Ankle Arthrodesis in Ankle Fractures Involving the Joint
}

\author{
Baris Yilmaz' ${ }^{1}$, Baran Komur ${ }^{2}$, Nurettin Heybeli ${ }^{3}$ \\ ${ }^{1}$ Orthopaedics and Traumatology Department, Fatih Sultan Mehmet Training and Research Hospital, Istanbul, \\ Turkey \\ ${ }^{2}$ Orthopaedics and Traumatology Department, Kanuni Sultan Suleyman Training and Research Hospital, \\ Istanbul, Turkey \\ ${ }^{3}$ Orthopaedics and Traumatology Department, Medical Faculty, Trakya University, Edirne, Turkey \\ Email: "barankomur@gmail.com
}

Received 21 April 2016; accepted 22 May 2016; published 27 May 2016

Copyright (C) 2016 by authors and OALib.

This work is licensed under the Creative Commons Attribution International License (CC BY).

http://creativecommons.org/licenses/by/4.0/

(c) (i) Open Access

\begin{abstract}
Posttraumatic osteoarthritis is a serious complication mostly involving weight bearing joints. It is not yet possible to predict which patients would develop posttraumatic osteoarthritis at a clinically relevant level. It is a common notion that, in comminuted fractures of the ankle joint that are highly likely to result in posttraumatic arthritis, interventions that aim to reduce the development of arthrosis should be attempted or achieving an ankle in an acceptable position for arthrodesis in the later periods should be targeted. Open type of fracture, a possible risk of infection and the duration of wound closure play a crucial role in the treatment of fracture, which is the main injury. In order to gain a better understanding of the importance of the treatment method to be chosen for the treatment of the trauma involving the ankle, a review of the information about ankle arthrodesis, which has an important place in the treatment of osteoarthritis, would be sufficient. We reviewed general principles and surgical approaches about this subject.
\end{abstract}

\section{Keywords}

Salvage Surgery, Ankle Arthrodesis, Ankle Fracture

Subject Areas: Orthopedics

\section{Introduction}

Posttraumatic osteoarthritis is a serious complication mostly involving weight bearing joints. This complication becomes more severe when the ankle is involved because the anatomic, biomechanical and cartilage characteristics of the ankle are different from those of other lower extremity joints [1] [2]. It is known that the ankle joint is

*Corresponding author. 
rarely affected from primary osteoarthritis and about $70 \%-80 \%$ of ankle osteoarthritis develops secondary to trauma [3]. The most common cause of posttraumatic ankle osteoarthritis is malleolar fractures with an incidence rate of 39\% [1]. In addition, pilon fractures are also an important risk for the development of posttraumatic arthritis [4] [5].

\section{Distribution and Evaluation}

It is not yet possible to predict which patients would develop posttraumatic osteoarthritis at a clinically relevant level. In addition, radiological degree of posttraumatic osteoarthritis may not always be associated with actual clinical symptoms like pain while walking and stiffness. The optimal timing of posttraumatic ankle osteoarthritis and the factors that affect the choice of the timing have not been elucidated yet [6]-[8]. On the other hand, osteoarthritis may develop particularly after ankle fracture dislocation independent of the duration or quality of reduction.

Within this context, the issue that should be discussed as a priority is which is the appropriate approach for an improperly treated fracture that is highly likely to result in osteoarthritis, whereas, the second issue to be focused on is how the treatment should be administered in case of development of complications such as osteoarthritis and/or nonunion.

Diagnosis can be made by X-rays, but sometimes CT can be helpful in order to investigate other bony deformities.

It is a common notion that, in comminuted fractures of the ankle joint that are highly likely to result in posttraumatic arthritis, interventions that aim to reduce the development of arthrosis should be attempted or achieving an ankle in an acceptable position for arthrodesis in the later periods should be targeted. Therefore, initial posttraumatic surgical planning should be performed accordingly [9]. On the other hand, another notion that has gained little acceptance is that primary arthrodesis should be performed in comminuted intraarticular fractures in which anatomic reduction could not be obtained and which are sure to develop arthrosis. Prognosis is poor if the injury left untreated, surgery can be considered when necessary [10].

\section{Treatment Options}

The choice between these two notions depends on taking consideration of patient and trauma-specific conditions. There may not be an appropriate method for the patient's ankle fracture and there may be some factors that prevent timely intervention.

The presence of clinically unstable patients, multitrauma patients or those in a life threating condition is an important determinant of damage control orthopaedics technique or its timing. Clinically unstable condition of the patient and the principles of damage control orthopaedics may delay the requirement of obtaining an anatomically stable fixation as early as possible. Since this situation will complicate obtaining anatomical restoration of the joint, the approach to be adopted will depend on the surgeon's level of experience.

Open type of fracture, a possible risk of infection and the duration of wound closure play a crucial role in the treatment of fracture, which is the main injury. In open fractures, debridement to be performed first for the prevention of infection and removal of dead tissues are of great importance. The wound should be closed primarily or using techniques such as skin grafting and flap surgery. It is important to make a good planning and, if a flap procedure is required, it should be performed in a way not to compromise possible future surgical interventions and not to interfere with routine activities of patient's daily life such as putting on shoes. If an infection has developed, a rapid and aggressive treatment should be initiated for infection. Even if the infection is confined to the soft tissues, the risk of osteomyelitis should be kept in mind [11] [Figure 1].

The presence of a systemic disease that is likely to compromise wound healing postoperatively affects the decision making for initial treatment. In such a case, surgical wound problems, which are likely to be followed by infection and necrosis, result in more unfavorable outcomes in the long term [Figure 2].

The tendency for delayed union and nonunion in ankle fractures is also an issue of great importance [12]. After trauma, anatomical integrity of the joint should be restored first, which subsequently should be supported with a strong fixation, and the grafting of the defect in the metaphyseal region, if necessary, is the treatment of choice for most intraarticular fractures. On the other hand, because of the possible risk of delayed union or nonunion of ankle fractures, these fractures should be closely followed up clinically and radiologically. If necessary, bone grafting can be repeated or the use of bone growth stimulators can be considered. If this situation is considered to be associated with the surgical implant performed, surgical revision may be required. The removal of 


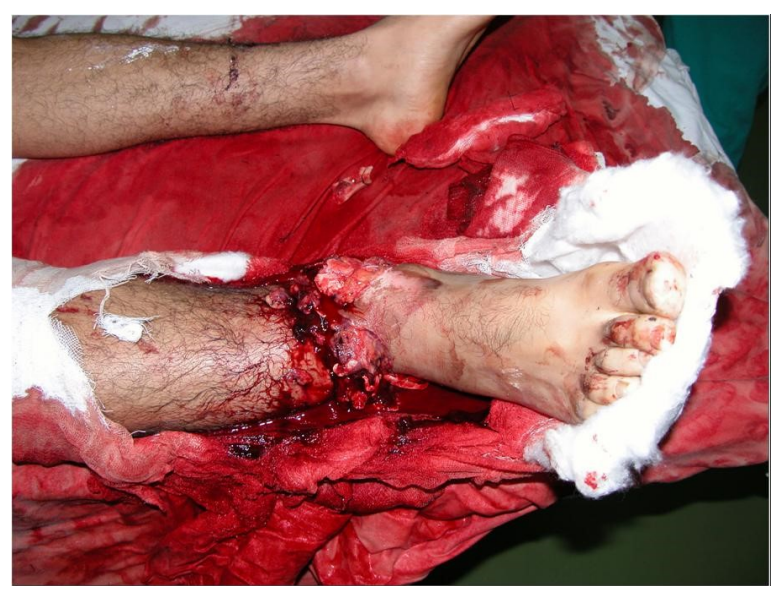

Figure 1. Patient with intraarticular open fracture of the ankle.

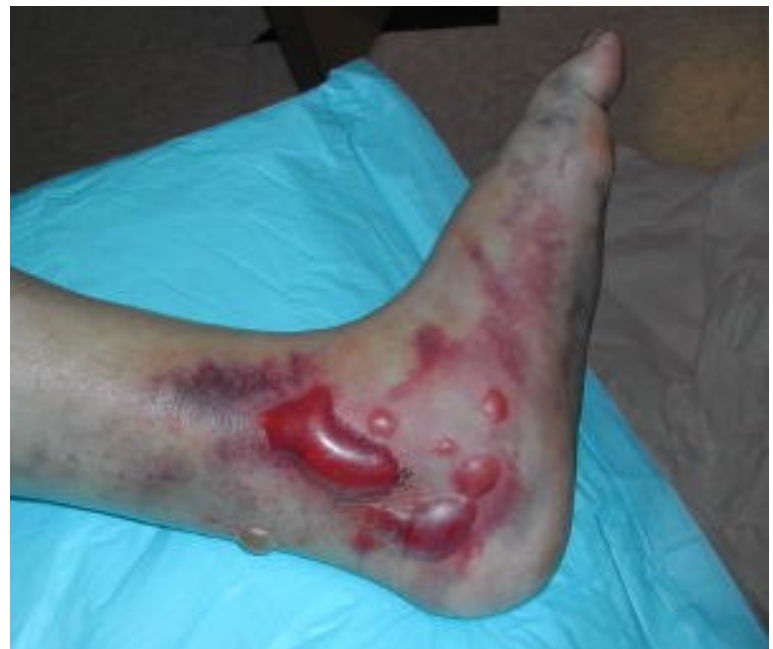

Figure 2. Skin problems are common in ankle fractures.

improper or instable internal fixation may be required or switched to another fixation technique. In case of nonunion, fibrous tissue at the fracture line is removed and cancellous bone grafting is performed to stimulate revascularization distally or proximally in an attempt to obtain bone stock.

In order to gain a better understanding of the importance of the treatment method to be chosen for the treatment of the trauma involving the ankle, a review of the information about ankle arthrodesis, which has an important place in the treatment of osteoarthritis, would be sufficient. Arthrodesis is the elimination of motion in a joint. The immobilization of the ankle with impaired function due to pain, and limitation of motion and contracture at the most appropriate position possible is a technique with a long history. This procedure eliminates the motion of the joint, however, with the relief of pain and the correction of the deformity resulting from contracture, functions are markedly improved [13].

Ankle arthrodesis is highly effective in pain control in the short and long term follow ups. On the other hand, a high rate of subtalar arthrosis and loss of tarsal mobility has been reported in long term follow up of tibiotalar arthrodesis [14]. Because of the risks such as the acceleration of arthrosis of neighboring joints, the generation of stress fractures and failure of arthrodesis, patients having undergone arthrodesis are not advised to do sports requiring running and jumping.

Arthrodesis was a common procedure before total joint arthroplasties have become popular. Arthrodesis of large joints such as the hip and knee joint causes a considerable restriction of the patient's functions. Since total joint arthroplasties performed in these joints have yielded satisfactory results, arthrodesis is only performed in ineligible patients for arthroplasty. In such a case, the indications for arthrodesis are as follows [12] [Table 1]. 
Table 1. Indications for arthrodesis.

PRIMARY ARTHRODESIS

- Infection

- Charcot arthropathy

- Arthritis of neighboring tissues [subtalar, talonavicular, calcaneocuboid, midfoot, knee]

- Deformity above or below the ankle joint [tibia, hindfoot, forefoot]

REVISION ARTHRODESIS

- Nonunion

- Malunion

- Infection [soft tissue or osteomyelitis]

- Complex regional pain syndrome [CRPS] or reflex sympathetic dystrophy [RSD]

- Subtalar arthritis

- Malleolar impingement

- Tendon problems

- Stress fracture

- Lymphedema

The clinical histories of this group of patients would help identify whether the etiology of arthritis is primary, posttraumatic, infectious, inflammatory or congenital. The association of the pain with the activity, whether it reliefs after resting or not, whether it comes at night or not, and the association of the source of pain with the joint may indicate a possible infection or tumor. The presence of diabetes mellitus, inflammatory arthritis, neuropathy or mental problems may be predictors of postoperative problems. In these patients, tobacco and alcohol use should also be taken into consideration [13].

Physical examination is an important part of decision making in these patients. The most important step is to identify the location and source of pain. In a patient with ankle arthritis, pain increases with dorsiflexion and plantar flexion. The increased pain with eversion and inversion may indicate an association with subtalar or talonavicular joint [15] [16]. If necessary, injection tests can be carried out for the establishment of diagnosis. The presence of a congenital deformity or one resulting from a previous trauma can be investigated as the cause of pain. Static and dynamic deformities of the lower extremity, examination of the range of motion of the hip and knee and leg-length inequality should be carefully evaluated. The overall appearance of the foot and previous cut or incision sites in case of revision, fusion or subsequent ankle surgery are important in planning. Previous skin graft or flap and planned incisions, the condition of the soft tissue as well as the flexibility of the skin should be carefully checked. Any signs of a systemic disease or infection [i.e. swelling, rash, previously drained wounds or fistulous tracts] should also be taken into consideration. Motor and sensory deficits of the foot and vascular problems should be assessed because the patient may have a neurovascular problem after trauma or surgery.

\section{Surgical Approaches and Fixation Techniques in Ankle Arthrodesis}

There are several surgical approaches for ankle arthrodesis. The incision site depends on the surgeon's preference. The patient's previous incisions, degree of deformity and the fixation technique influence the surgeon's preference [Figure 3].

Arthroscopic approach is recommended in patients with minimal deformity and soft tissue compromise. To minimize the complications, arthroscopy should be performed via three portals without skeletal traction. It is not usually used for revision arthrodesis [17]. Mini-arthrotomy requires minimal soft tissue dissection and a good visualization. It is the most common surgical technique which requires less technical equipment compared to that in arthroscopic technique. This technique is more appropriate for primary arthrodesis, though not for patients with deformities or bone loss or in case of revision.

The anterior approach is most commonly used for ankle arthroplasty. The interval for an anterior approach is between the anterior tibial tendon and extensor tendon with retraction of the neurovascular bundle. Even though 


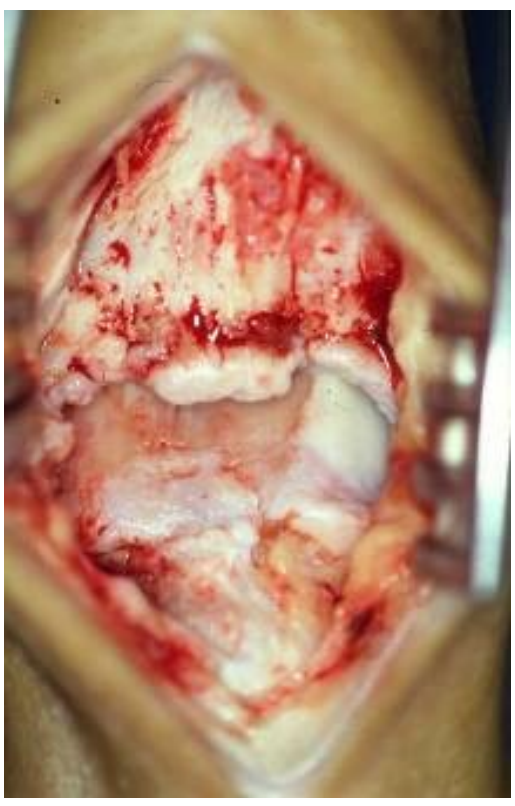

(a)

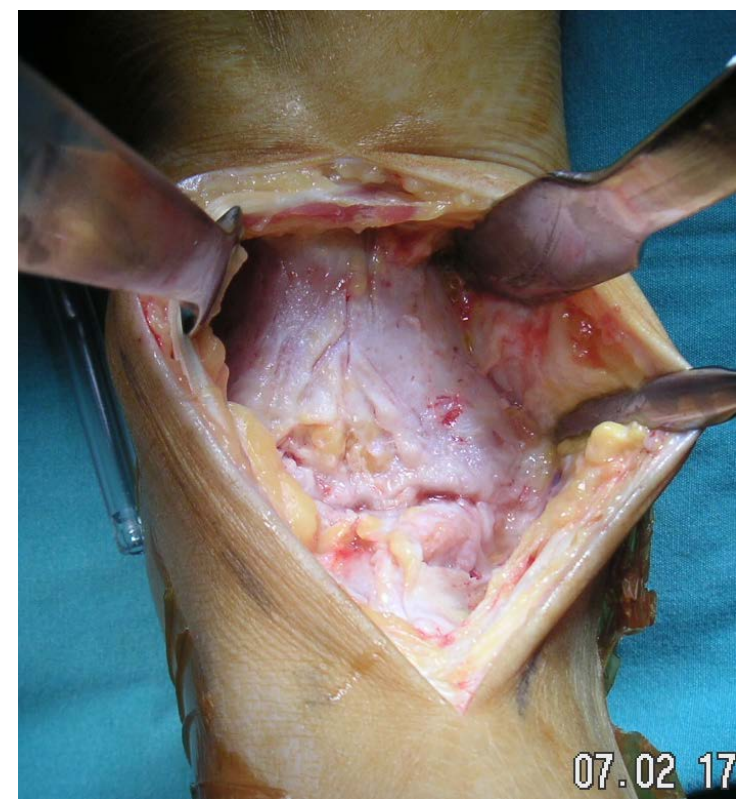

(b)

Figure 3. Clinical image of the patient with ankle osteoarthritis during surgery.

this approach provides a very good exposure to the ankle, it has been reported to be associated with deep and superficial peroneal neuromas and common wound healing complications. For complicated and revisional arthrodesis, a lateral transfibular incision is preferred. After a lateral incision is made and the distal fibula is elevated, a perfect visualization of the ankle and subtalar joint is obtained. The disadvantages of this approach include the impairment of the normal anatomy of the ankle and limited medial visualization.

Achilles tendon gap is used to reach the ankle using a posterior incision. This incision is more useful in hindfoot fusions [12]. This approach is also preferred in scar formation due to previous infections, poorly healed incisions or soft-tissue related problems resulting from open fracture in the anterior aspect. A fusion rate of $80 \%$ $100 \%$ has been reported with open, mini-open or arthroscopic techniques in modern series [18]. Arthroscopic techniques, being associated with lower rates of morbidity and more rapid achievement of fusion, are advantageous. On the other hand, arthroscopic techniques are inferior to open techniques in terms of correcting severe deformities and restoring bone loss [17]. Various fixation materials are utilized to achieve ankle fusion [19]. Cannulated screws are commonly used for fixation in primary fusion. Multiple screws can be inserted correctly using fluoroscopy [Figure 4 and Figure 5]. Plates and screws are usually employed in patients with complicated fusion, bone loss and deformities [20] [21]. They are also used when the fusion is extended to include the subtalar joint in fusion. The main disadvantage is that it requires a wide dissection for the use of plates. Orthopaedic staples, Kirschner wire and cerclage wires also have a place in ankle fusion. In arthrodesis performed using intramedullary screws, the subtalar joint is also included in arthrodesis; these patients usually have a severe deformity of the talus and a considerable bone loss. External fixators, particularly ring fixators, are useful for ankle fusion and used in patients with infection, bone loss or deformities [22]. At the same time, they offer advantages such as the correction of deformities and leg-length inequality and use in patients with bone defects.

The ideal position for ankle arthrodesis is neutral flexion, external rotation of $5^{\circ}-10^{\circ}, 5^{\circ}$ of valgus and with the talus placed posterior to the tibia. There are a number of proven techniques for fusion but all have basic principles in common [23]. The surgical techniques offering minimum bone loss, surfaces that will ensure correction of deformities, and minimum soft tissue injury are preferred. All cartilage and fibrous tissues are removed, thus exposing the healthy, vascularized bone. Fluoroscopy should be used to ensure proper placement of the implant chosen. If necessary, lengthening of the Achilles tendon, gastrocnemius slide or tendon transfer can be performed. A number of studies have reported adequate rates of ankle fusion without the use of allograft or bone graft. On the other hand, bone grafting is advised in patients with tobacco use, a history of infection, previous open injury, neuropathy, spasticity, pilon fractures, osteonecrosis of the talus and severe health problems. Bone growth stimulators can also be used to increase the fusion rates. Several studies have shown an association 
between electric current and new bone growth [12]. One of the options is the use of ultrasound to improve bone healing, which should be considered when a complex fusion is required and in patients with risk factors for nonunion.

Simple fusion is preferred in patients with minimal deformity and bone loss. Mini-arthrotomy is the most recommended technique requiring anatomical bone cut and the placement of two-four [preferably three] large cannulated screws [12] [Figure 4 and Figure 5]. Deformity should be identified in patients with severe ankle deformities and should be addressed as a priority before ankle fusion. In such cases, an anterior approach can be employed, whereas, a lateral approach is usually preferred in more severe deformities. In order to bring the leg back to normal axis, additional procedures may be required [calcaneous, supramalleolar or metatarsal osteotomy]. If a flat bone surface can be achieved without causing many defects, cannulated screws or plate screws can be used. If the defect is severe, causing shortening of the leg, the correction of deformity can be achieved by using bone grafts and the shortening of the leg can be avoided. Plates and screws are used to stabilize the fusion by keeping the bone graft in a safe position, in which case the use of a cannulated screw is not advised [18] [19].

The main reasons of nonunion or malunion should first be investigated. The three main reasons include technical error [surgeon], noncompliance [patient] and local or systemic problems [12]. In case of a technical error, the available fixation should be assessed, be removed or revised, if necessary, and autografts and cannulated screws and postoperative bone stimulators can be used, like in primary fusion. Noncompliant patients should be warned about tobacco use, their postoperative expectations and the risk of nonunion. Bone grafts and stimulators can also be utilized in these patients. The infection status regarding local and systemic problems, soft tissue problems and systemic diseases should be addressed.

In some patients, a previous or current infection should be resolved before obtaining a solid fusion. Infection should be treated in a stepwise fashion; the first step is the debridement of the infected bone and soft tissues and the use of antibiotic impregnated beads for the area. Subsequently, a 6-week treatment with intravenous antibiotics is required. If the infection is adequately resolved, laboratory tests are run and then fusion is performed. In such a case, an external fixator is usually preferred. An external fixator can also be a good alternative for the correction of a defect resulting from surgical debridement [24] [25].

An MRI and/or a CT can be performed to determine osteonecrosis of the talus and subtalar joint involvement. In case of a talar bone loss, ankle fusion can be performed using the methods described above, if the subtalar joint has not been affected. If the subtalar joint has also been affected, the recommended procedure is tibiotalocalcaneal fusion [Figure 6] [26].

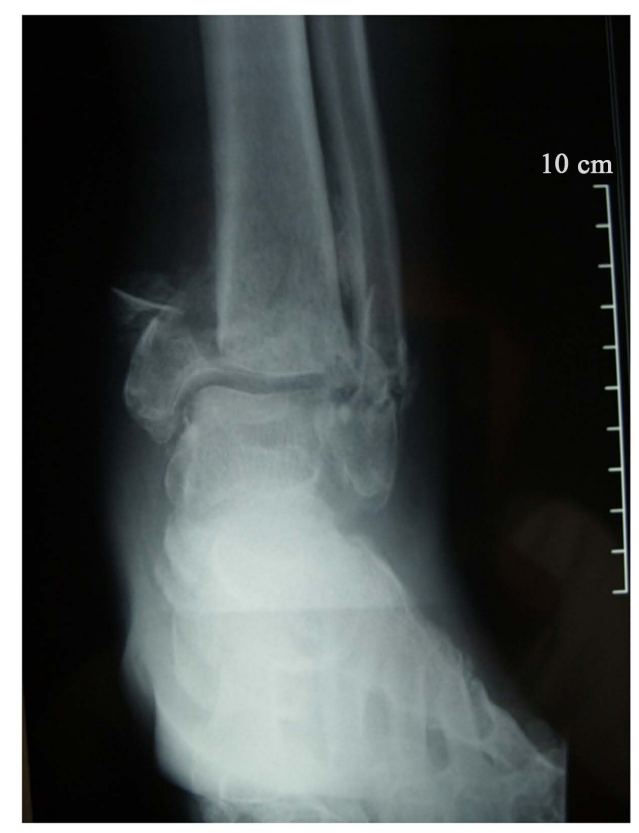

(a)

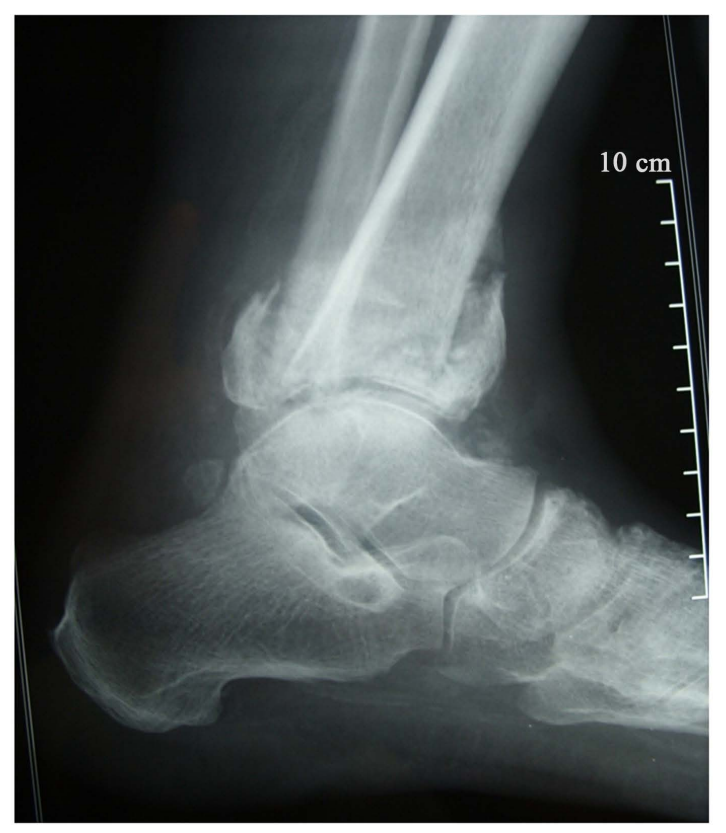

(b)

Figure 4. Delayed union and nonunion are common in fractures of the ankle involving the joint. 


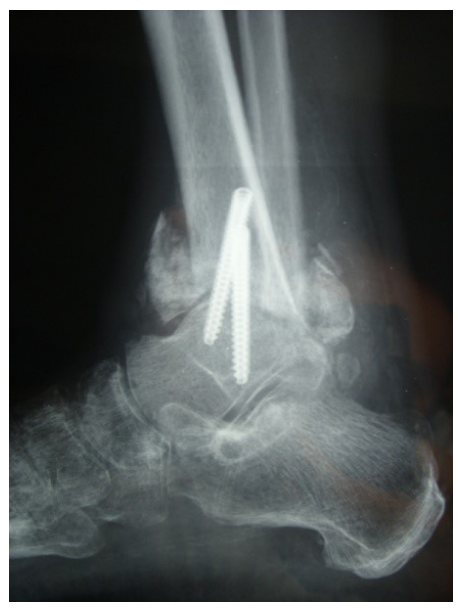

(a)

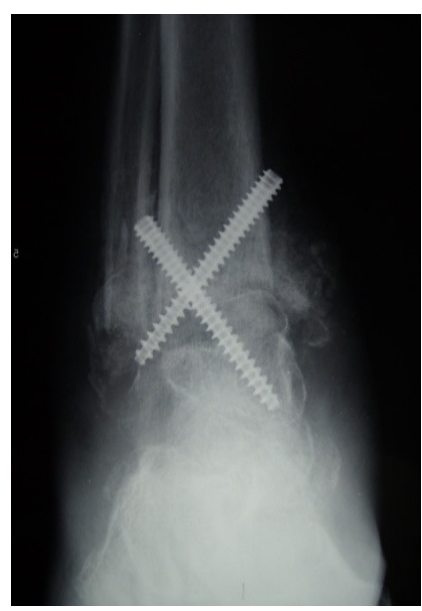

(b)

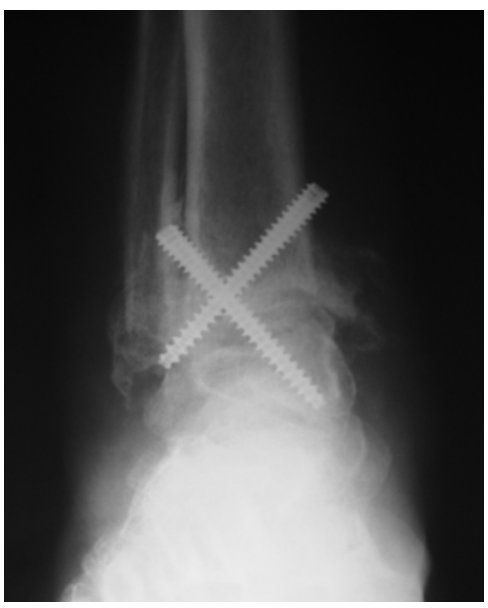

(c)

Figure 5. Postoperative early period (a) lateral; (b) AP; (c) late period AP radiograph of the patient undergoing ankle arthrodesis with internal fixation.

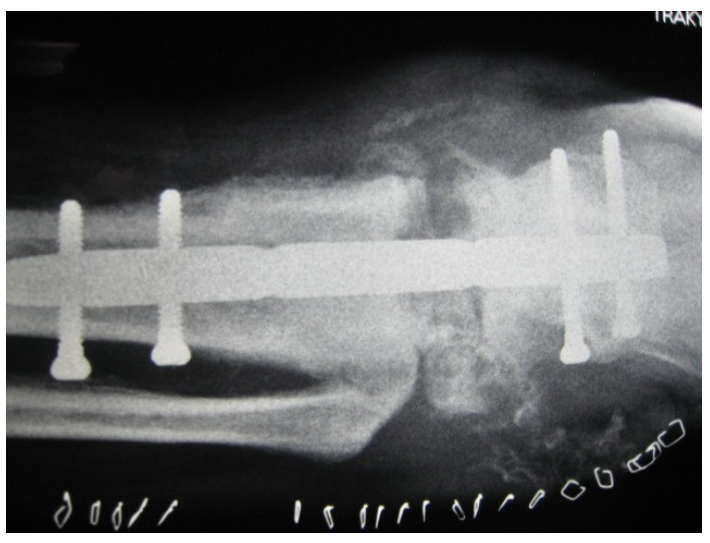

(a)

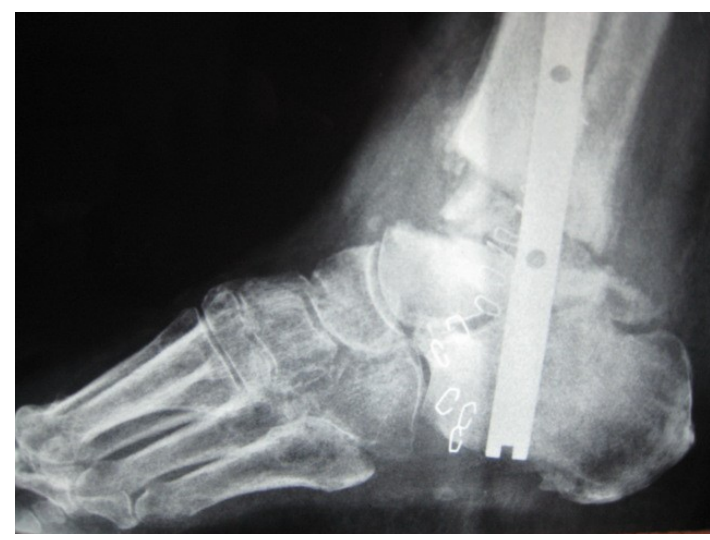

(b)

Figure 6. Postoperative (a) AP; (b) lateral radiographs of the patient in whom tibiotalocalcaneal intramedullary nails were placed.

\section{Conclusion}

The performance of ankle fusion after failed ankle arthroplasty includes difficulties such as large amount of bone loss, the possibility of subtalar joint involvement, infection and correction of leg-length inequality. Since there will be at least $2 \mathrm{~cm}$ of bone loss, first bone grafting can be placed and then shoe modification can be used to compensate for the difference in length. As long as an autograft [iliac] or an Ilizarov-type device is not used for leg lengthening, the defect will result in an apparent leg-length inequality. Prior to this procedure, it is of great importance to discuss the possibility of leg-length inequality with the patient. In case of an infection, it is recommended first to remove the implant as described above, to treat the infection, and then to plan for performing fusion.

\section{References}

[1] Valderrabano, V., Horisberger, M., Russell, I., Dougall, H. and Hintermann, B. (2009) Etiology of Ankle Osteoarthritis. Clinical Orthopaedics and Related Research, 467, 1800-1806. http://dx.doi.org/10.1007/s11999-008-0543-6

[2] Michelson, J. (2006) Lower Extremity Considerations: Foot and Ankle In: Moskowitz, R.W., Altman, R.D., Hochberg, M.C., Buckwalter, J.A. and Goldberg, V.M., Eds., Osteoarthritis, 4th Edition, Lippincott Williams \& Wilkins, Philadelphia, 215-226.

[3] Thomas, R.H. and Daniels, T.R. (2003) Ankle Arthritis. Journal of Bone and Joint Surgery American Volume, 85, 923- 
936.

[4] Sands, A., Grujie, L., Byck, D.C., et al. (1998) Clinical and Functional Outcomes of Internal Fixation of Displaced Pilon Fractures. Clinical Orthopaedics and Related Research, 347, 131-137. http://dx.doi.org/10.1097/00003086-199802000-00016

[5] Valderrabano, V., Nigg, B.M., von Tscharner, V., Stefanyshyn, D.J., Goepfert, B. and Hintermann, B. (2007) Gait Analysis in Ankle Osteoarthritis and Total Ankle Replacement. Clinical Biomechanics, 22, 894-904. http://dx.doi.org/10.1016/j.clinbiomech.2007.05.003

[6] Witteveen, A.G., Giannini, S., Guido, G., Jerosch, J., Lohrer, H., Vannini, F., et al. (2008) A Prospective Multi-Centre, Open Study of the Safety and Efficacy of Hylan G-F 20 [Synvisc] in Patients with Symptomatic Ankle [Talo-Crural] Osteoarthritis. Foot and Ankle Surgery, 14, 145-152. http://dx.doi.org/10.1016/j.fas.2008.01.001

[7] Saltzman, C.L., Salamon, M.L., Blanchard, G.M., Huff, T., Hayes, A., Buckwalter, J.A., et al. (2005) Epidemiology of Ankle Arthritis: Report of a Consecutive Series of 639 Patients from a Tertiary Orthopaedic Center. Iowa Orthopaedic Journal, 25, 44-46.

[8] Heybeli, N., Ozcan, M. and Yalnız, E. (2008) Ankle Stiffness and Osteoarthritis in Fracture-Dislocation: An Avoidable Complication or Natural History. Archives of Orthopaedic and Trauma Surgery, 128, 639-640. http://dx.doi.org/10.1007/s00402-008-0578-X

[9] Cox, F.J. (1965) Fractures of the Ankle Involving the Lower Articular Surface of the Tibia. Clinical Orthopaedics and Related Research, 42, 51-55. http://dx.doi.org/10.1097/00003086-196500420-00006

[10] Mast, J.W., Spiegel, P.G. and Pappas, J.N. (1988) Fractures of the Tibial Pilon. Clinical Orthopaedics and Related Research, 230, 68-82. http://dx.doi.org/10.1097/00003086-198805000-00008

[11] Eralp, L., Kocaoglu, M. and Rashid, H. (2007) Reconstruction of Segmental Bone Defects Due to Chronic Osteomyelitis with Use of an External Fixator and an Intramedullary Nail. Surgical Technique. Journal of Bone and Joint Surgery American Volume, 89, 183-195. http://dx.doi.org/10.2106/JBJS.F.01579

[12] Nunley, J.A., Pfeffer, G.B., Sanders, R.W. and Trepman, E. (2002) Advanced Reconstruction, Foot and Ankle. American Orthopaedic Foot and Ankle Society, 181-189.

[13] Gumann, G. (2004) Fracture of the Foot and Ankle. Elseiver Saunders, 373-402.

[14] Coester, L.M., Saltzman, L.C., Leupold, J. and Pontarelli, W. (2001) Long Term Results Following Ankle Arthrodesis for Post-Traumatic Arthritis. Journal of Bone and Joint Surgery American Volume, 83, 219-228.

[15] Kile, T.A. and Alford, D.W. (1998) Arthritis and Deformities of the Hindfoot and Ankle. In: Mizel, M.S., Miller, R.A. and Scioli, M.W., Eds., Orthopaedic Knowledge Update, American Academy of Orthopaedic Surgeons, Rosemont, 279-291.

[16] Khazzam, M., Long, J.T., Marks, R.M. and Harris, G.F. (2006) Preoperative Gait Characterization of Patients with Ankle Arthrosis. Gait \& Posture, 24, 85-93. http://dx.doi.org/10.1016/j.gaitpost.2005.07.006

[17] Jerosch, J. (1999) Arthroscopic Ankle Surgery: Indications, Methods, Results, Complications. Der Orthopäde, 28, 538549.

[18] Helm, R. (1990) The Results of Ankle Arthrodesis. The Bone \& Joint Journal, 72, 247-252.

[19] Dennis, D.A., Clayton, M.L., Wong, D.A., Marc, R.P. and Susman, M.H. (1990) Internal Fixation Compression Arthrodesis of the Ankle. Clinical Orthopaedics and Related Research, 253, 212-220.

[20] Mohamedean, A., Said, H.G., El-Sharkawi, M., El-Adly, W. and Said, G.Z. (2009) Technique and Short-Term Results of Ankle Arthrodesis Using Anterior Plating. International Orthopaedics, 34, 833-837.

[21] Plaass, C., Knupp, M., Barg, A. and Hintermann, B. (2009) Anterior Double Plating for Rigid Fixation of Isolated Tibiotalar Arthrodesis. Foot \& Ankle International, 30, 631-639. http://dx.doi.org/10.3113/FAI.2009.0631

[22] Kovoor, C.C., Padmanabhan, V., Bhaskar, D., George, V.V. and Viswanath, S. (2009) Ankle Fusion for Bone Loss around the Ankle Joint Using the Ilizarov Technique. The Bone \& Joint Journal, 91, 361-366. http://dx.doi.org/10.3113/FAI.2009.0631

[23] Bacin, C.C. (1986) A Simple Technique for Arthrodesis of the Ankle. The Bone \& Joint Journal, 68, 266-267.

[24] Colgrove, R.C. and Bruffey, J.D. (2001) Ankle Arthrodesis: Combined Internal-External Fixation. Foot \& Ankle International, 22, 92-97.

[25] Bernan, A.T., Bosacco, S.J., Parks, B.G., et al. (1999) Compression Arthrodesis of the Ankle by Triangular External Fixation: Biomechanical and Clinical Evaluation. Orthopedics, 22, 1129-1134.

[26] Goebel, M., Muckley, T., Gerdesmeyer, L., Militz, M. and Buhren, V. (2003) Die kombinierte Kompressionsmarknagelarthrodese von OSG und USG. Der Unfallchirurg, 106, 633-641. http://dx.doi.org/10.1007/s00113-003-0626-5 